P196 (continued)

Evaluation: An online survey was sent to listserv subscribers $(n=332)$. Survey completion incentive was entry into a random $\$ 25$ gift card drawing. Survey respondents $(n=96)$ self-reported changes. Approximately 70\% used newsletters one to three times a month and said they were helpful in the following ways: provided reliable, trusted information (68\%), generated new programming ideas $(57 \%)$, helped children try new foods (52\%), provided tips for feeding children (45\%), increased MyPlate food groups at meals and snacks (30\%), provided social media topic ideas (28\%), and increased work efficiency/ productivity (25\%).

Conclusions and Implications: Utilizing an online format for sharing recipes is a productive vehicle to provide feeding and healthy eating tips and strategies to audiences. Building on the interest of users by offering new, healthy, and tasty recipes for children provides a means for increasing awareness of and action toward more desirable behaviors.

Funding: None

\section{P197 Nutrition Education to Increase the Self-Efficacy of Low-Income Children to Make Healthy and Safe Food Choices}

Lynda Zimmerman, MS, RD, LD, zimmermanl@missouri. edu, University of Missouri Extension, 105 East 5th Street, Kansas City, MO 64106; K. Keller, PhD, CFLE

Objective: To help youth make healthful and safe food choices.

Target Audience: The target audience was low-income, SNAP-Ed eligible children (ages 7-11 years) attending an after-school program.

Theory, Prior Research, Rationale: This program is based on Social Cognitive Theory (SCT). Activities and discussions were designed to help increase children's selfefficacy, a core construct of SCT.

Description: The program was adapted from University of Missouri Extension's Kids in the Kitchen, a researchbased curriculum shown to improve youth dietary quality and food preparation skills. The program consisted of 5 , 45-minute, weekly sessions. Each session included a food safety mini-lesson, preparation of a simple recipe, and discussion, including food groups represented the ingredients used, possible substitutions for ingredients, how children might ask an adult to purchase ingredients not typically found in their homes, and safe storage of ingredients and the food made if there were leftovers. To encourage active participation, adult leaders worked with small groups of 5-6 children.

Evaluation: Thirty-seven youth participated in the program, divided into groups by grades $2-3$ and $4-5$. Pre- and post-assessments measured food safety knowledge, healthful food requests at home, and eating habits. Paired t-tests, pre- and post-program, revealed improved food safety knowledge and eating habits; healthful food requests increased for some but not all indicators. Student age had no effect. Parents completed a post-program questionnaire that showed improved ratings in program-related changes in children's knowledge and cooking skills. Parents also provided examples of children's new skills and the program's benefits.

Conclusions and Implications: A cooking program presented in five weekly sessions in an after-school setting is effective in helping low-income youth increase food safety knowledge and healthful eating habits.

Funding: USDA

\section{P198 Interagency Collaboration to Develop a Healthy Pantry Guide for the State of Washington}

Alexandra Bush-Kaufman, MPH, RD, alexandra.kaufman@ wsu.edu, Washington State University Extension, Pullman, WA 99163; L. Woodworth, MSN, RDN, Thurston County Food Bank; C. Huskey, RD, Washington State Department of Health; J. Wells, MPH; K. Barale, MS, RD, FADA, Washington State University Extension; M. Catalina Aragón, MS; J. Armstrong Shultz, PhD

Objective: Develop a "healthy pantry" strategies and actions guide through an interagency collaboration for use by local SNAP-Ed implementing agencies (IA), subcontractors and food pantry managers to improve the emergency food environment.

Target Audience: SNAP-Ed implementers and their food pantry partners.

Theory, Prior Research, Rationale: SNAP-Ed IAs often work closely with food pantries and other emergency food outlets. The food pantry environment plays a critical role in client diet. Washington SNAP-Ed IAs-State Department of Health and Washington State University Extension-identified a need for strategies and an assessment tool to support environmental change within the food pantry setting.

Description: Washington State (WA) IAs engaged anti-hunger stakeholders for content review of the tools developed. Shared goals were established with program objectives designed to meet SNAP-Ed Western Region Framework metrics. WA IAs and members of Washington's anti-hunger community met weekly or monthly, in-person and via conference call, from June 2015 to February 2016. Evaluation: The workgroup conducted content analysis of success stories collected by the WA Food Coalition, an advocacy group; reviewed and incorporated best practices from the literature; edited strategies for allowable SNAPEd practices; and conducted peer review and cognitive pretesting with pantry partners and WA State Department of Agriculture staff.

Conclusions and Implications: Results of this workgroup include: a rationale narrative, a draft self-assessment tool for pantry use, and a "healthy pantry" strategy guide to address actions areas. These efforts enhance the environmental change work of WA SNAP-Ed with food pantry partners. Significant planning is necessary to overcome geographic and organizational barriers and to facilitate interagency collaboration.

Funding: Supplemental Nutrition Assistance Program Education 